\title{
On the use of structural equation models in experimental designs: Two extensions
}

\author{
Richard P. Bagozzi, Youjae Yi \\ and Surrendra Singh * \\ School of Business Administration, The University of Michigan, \\ Ann Arbor, MI 48109-1234, USA
}

\begin{abstract}
Bagozzi and Yi (1989) recently introduced new procedures for using structural equation models in experimental designs with L.ISkEL. We extend their research by showing that the structural equation analysis of experimental designs can be accomplished via Wold's partial least squares (PLS) approach, which can be used without many of the assumptions necessary for maximum likelihood estimation in LISREL. We show that PLS is applicable not only to the basic design, but also to other complex designs. We also identify two restrictive assumptions implicit in Bagozzi and Yi's step-down analysis procedures, and describe a more general approach that can be used even when these assumptions are not met. The proposed procedures are illustrated with Bagozzi and Yi's data, and the conditions suitable for alternative procedures are discussed.
\end{abstract}

\section{Introduction}

Bagozzi and Yi (1989) recently introduced new procedures for the analysis of experimental data especially in Manova designs (see also Kühnel, 1988). They described the analytic procedures for various experimental designs with the widely used computer program LISREL (Jöreskog and Sörbom, 1986). Given the frequent use of experimental designs and the popularity of LISREL in marketing, their

\footnotetext{
* The authors thank the editor and anonymous $I J R M$ reviewers for their helpful comments on a previous version of this article. The financial assistance of The University of Michigan's School of Business Administration is also gratefully acknowledged.
}

Intern. J. of Research in Marketing 8 (1991) 125-140

North-Holland procedures can be potentially useful to marketing rescarchers (Yi, 1990).

Although Bagozzi and Yi's (1989) procedures provide a powerful means for analyzing experimental data, the use of their procedures might be limited for several reasons. First, experimental data often do not satisfy the requirements of maximum likelihood estimation in LISREL such as multivariate normality, interval scaling, and large sample sizes. Also, improper or non-convergent solutions sometimes occur in LISREL analyses, which will reduce the interpretability of estimates (e.g., Gerbing and Anderson, 1987). It would be desirable to have an alternative procedure for analyzing such data to which LISREL is not well suited. Second, Bagozzi and Yi's procedures for the step-down analysis, one particular type of MANOVA, make two implicit assumptions: (1) variances and covariances of dependent variables are equal across groups, and (2) causal paths among dependent variables are invariant across groups. When these assumptions are violated, their step-down analysis procedures are not appropriate. In fact, as shown below, the first assumption is invalid for Bagozzi and Yi's data. Thus, we need more general procedures which do not rely upon such restrictive assumptions.

The purpose of the present paper is to extend Bagozzi and Yi's (1989) research in these two respects. First, we demonstrate that the structural equation analysis of MANOVA designs can be accomplished via Wold's (1985) partial least squares (PLS) approach, which avoids many of the assumptions and chances that improper solutions will occur in LISREL analyses. We show that PLS is applicable not only to the basic design, but also to other MANOVA designs (e.g., latent variable MANOVA, 
step-down analysis). Second, we suggest an alternative procedure for step-down analyses which does not require the two restrictive assumptions implicitly made in Bagozzi and Yi's procedures. We close with a discussion of the conditions when one procedure might be preferred over the other. Throughout the paper, the proposed procedures are illustrated with the data used in Bagozzi and Yi's (1989) original article.

\section{MANOVA with PLS}

\subsection{LISREL formulation of MANOVA}

To demonstrate the use of structural equation models for the analysis of experimental data, we use MANOva designs with three dependent variables $\left(Y_{1}, Y_{2}, Y_{3}\right)$ and two groups (experimental and control groups). Fig. 1A illustrates the LISREL specification for this de-

\section{A. LISREL Specification}

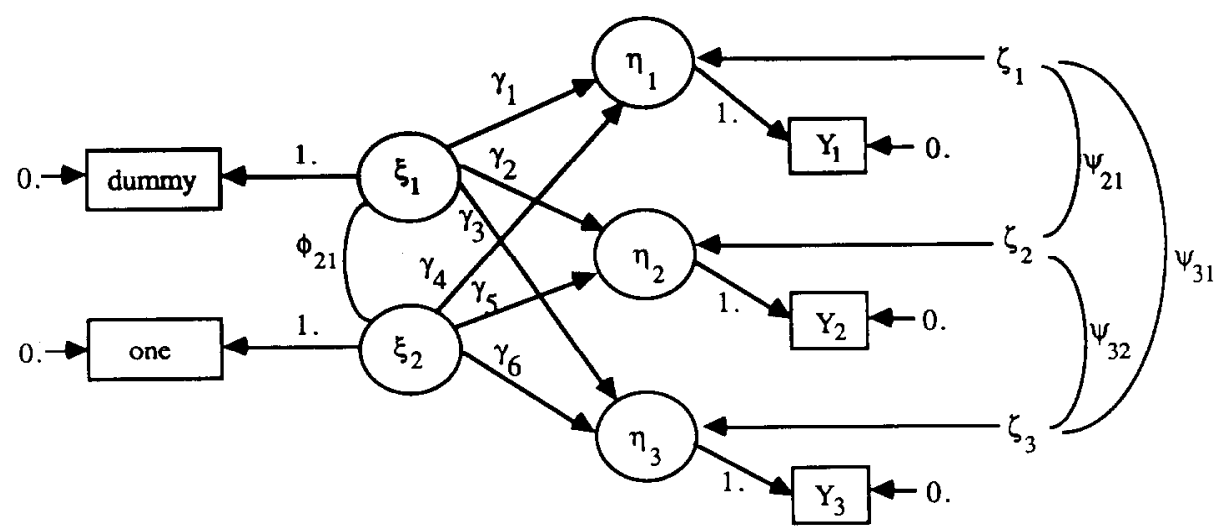

B. PLS Specification

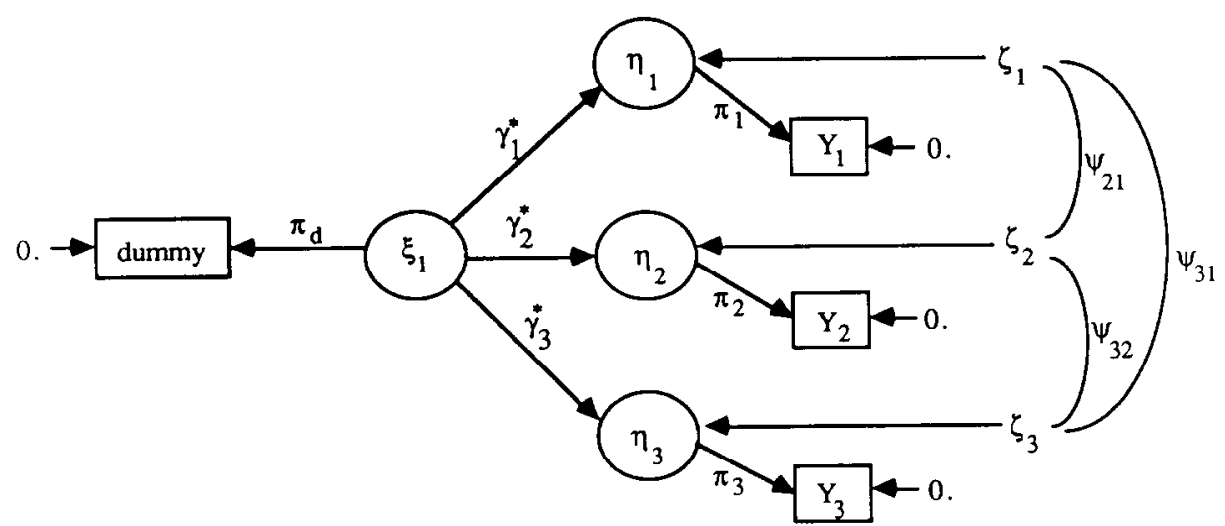

Fig. 1. LISREL and PLS models for MANOva for three dependent variables (for PLS specification, see Appendix A). 
sign (see Bagozzi and Yi, 1989, p. 273). There are two parts in the specification: measurement and structural models.

(a) Measurement model:

Dummy $=\xi_{1}$,

One $=\xi_{2}$,

$Y_{i}=\eta_{i} \quad$ for $i=1,2,3$,

where Dummy $= \begin{cases}0, & \text { control group, } \\ 1, & \text { experimental group. }\end{cases}$

(b) Structural model:

$\eta_{i}=\gamma_{i} \xi_{1}+\gamma_{i+3} \xi_{2}+\xi_{i}$ for $i=1,2,3$.

Note that the dummy variable is an exogenous latent variable $\left(\xi_{1}\right)$ that represents two groups: control and experimental groups. There is a single indicator with a fixed loading of unity and no corresponding residual. Also note that a pseudo-variable (i.e., "One") is used as another exogenous latent variable $\left(\xi_{2}\right)$ to capture the means or locations of dependent variables. Because the "Dummy" variable is 0 for the control group and 1 for the experimental group, the paths (i.e., $\gamma_{4}, \gamma_{5}$, $\gamma_{6}$ ) from the "One" latent variable to dependent variables correspond to the means of dependent variables for the control group, and the paths (i.e., $\gamma_{1}, \gamma_{2}, \gamma_{3}$ ) from the dummy variable to dependent variables reflect the differences in their means across the two groups. For example, the means of $Y_{1}$ are $\gamma_{4}$ and $\left(\gamma_{1}+\gamma_{4}\right)$ for the control and experimental groups, respectively, and thus $\gamma_{1}$ is the mean difference in $Y_{1}$ between the two groups. The significance of the mean differences can be tested either individually with the critical ratios ( $t$-values) for the parameters or globally with the chi-square difference tests of the zero restrictions for these parameters (Bagozzi and $\mathrm{Yi}, 1989$ ).

\subsection{General PLS model}

Before we present the PLS formulation of MANOVA, we present a brief overview of the general PLS model, including specification and estimation. Section 5 considers the assumptions made in the PLS model and compares it with LISREL. For more details, see Wold (1982, 1985).

Specification. Relations among latent variables are expressed in the following system of equations:

$\eta=\beta_{0}+\beta \eta+\nu$,

where $\eta$ is a vector of latent variables, $\beta_{0}$ is a location parameter vector, $\beta$ is a matrix of coefficients relating the $\eta$ 's among themselves, and $\nu$ is a vector of residuals for the $\eta$ 's. Equation ( 1 ) is sometimes referred to as the theoretical relations or the structural equation model. Latent variables are connected to observations through the following system of equations:

$\boldsymbol{y}=\Pi_{0}+\Pi \eta+\varepsilon$,

where $y$ is a vector of manifest variables, $\Pi_{0}$ is a location parameter vector, $\Pi$ is a matrix of loading coefficients (analogous to factor loadings), and $\varepsilon$ is a vector of residuals for the $\boldsymbol{y}$ 's. Equation (2) is usually referred to as the measurement model. It is assumed that $\operatorname{Cov}(\nu, \varepsilon)=0$.

The covariance matrix for the $\eta$ 's is written as

$P=(I-\beta)^{-1} \Psi\left(I-\beta^{\prime}\right)^{-1}$,

where $I$ is an identity matrix and $\Psi=\operatorname{Cov}(\nu)$. For the $y$ 's, the covariance matrix is

$\Sigma=\Pi P \Pi^{\prime}+\theta$,

where $\theta=\operatorname{Cov}(\varepsilon)$.

Estimation. To facilitate the discussion of estimation, the following equation is added to equations (1) and (2):

$\eta=\boldsymbol{\Omega}+\boldsymbol{\delta}$,

where $\Omega$ is a matrix of coefficients making latent variables dependent upon manifest variables, and $\delta$ is a vector of residuals. Estimation under the PLS algorithm then proceeds in three steps. In the first, iterative 
estimations of $\Omega$ are performed. This consists of a sequence of ordinary least squares (oLs) regressions, linear operations, and square root extractions. In the second step, $\beta$ and $\pi$ are estimated non-iteratively using the latent variables estimated in the first step. This is done assuming that the location parameters are zero. Finally, in the third step, the location parameters and generative relations are estimated. This is done non-iteratively with oLS regressions.

More intuitively, the PLS program proceeds iteratively by first estimating each latent variable from its observed indicators and then refining it by relating each latent variable to other latent variables' indicators. Once the latent variables have been estimated, they are then correlated, and the structural parameters of the model are estimated via path analysis using oLs regression. The resulting coefficients are interpreted as standardized partial regression coefficients.

\subsection{PLS formulation of MANOVA}

We propose that MANOVA designs can also be analyzed with PLS. Fig. 1B shows the specification of the PLS model that is equivalent to the LISREL model in Fig. 1A. Like the LISREL model, the PLS model has two parts:

(a) Measurement model:

Dummy $=\pi_{d} \xi_{1}$,

$Y_{i}=\pi_{i} \eta_{i} \quad$ for $i=1,2,3$.

(b) Structural model:

$\eta_{i}=I_{i}+\gamma_{i}^{*} \xi_{1}+\zeta_{i}^{*} \quad$ for $i=1,2,3$,

where $I_{i}$ is the intercept that reflects the location of the dependent variable $\left(\eta_{i}\right)$. To make the comparisons of our LISREL and PLS formulations of MANOVA as simple as possible, we have made several redefinitions of variables and parameters found in Wold's $(1982,1985)$ original exposition. Namely, $\beta_{0 i}$ $=I_{i}, \beta_{1 i}=\beta_{2 i}=\beta_{3 i}=0, \beta_{4 i}=\gamma_{i}^{*}, \eta_{4}=\xi_{1}$, and $\nu_{i}=\zeta_{i}^{*}$.
We can note two differences between PLS and LISREL specifications. First, the pseudovariable of one is not used in the PLS specification, whereas it is necessary in the LISREL specification. This is because PLS estimates the location parameters as intercepts without the need for introducing such a pseudo-variable. Second, the loadings relating latent variables to observed measures are set free and estimated in the PLS formulation, whereas they are fixed to unity in the LISREL formulation. For example, the loadings (i.e., $\pi_{i}$ ) for endogenous variables are free for PLS but fixed to 1.0 for LISREL. The path coefficients (i.e., $\gamma_{1}^{*}$, $\gamma_{2}^{*}, \gamma_{3}^{*}$ ) from the dummy latent variable to endogenous variables can be examined in order to test whether the means are significantly different across groups.

We will now show the equivalence of the LISREL and PLS models by combining the measurement and structural parts of each model. This will permit us to compare the parameters of one model with those of the other.

LISREL model:

$$
\begin{aligned}
Y_{i}= & \eta_{i} \\
= & \gamma_{i} \xi_{1}+\gamma_{i+3} \xi_{2}+\zeta_{i} \\
& \quad \text { ince } \eta_{i}=\gamma_{i} \xi_{1}+\gamma_{i+3} \xi_{2}+\xi_{i} \\
= & \gamma_{i+3}+\gamma_{i} \text { Dummy }+\zeta_{i} \\
\quad & \text { since } \xi_{1}=\text { Dummy, } \xi_{2}=1.0 . \\
& \text { PLS model: }
\end{aligned}
$$

$$
\begin{aligned}
Y_{i}= & \pi_{i} \eta_{i} \\
= & \pi_{i}\left(I_{i}+\gamma_{i}^{*} \xi_{1}+\zeta_{i}^{*}\right) \\
& \quad \text { since } \eta_{i}=I_{i}+\gamma_{i}^{*} \xi_{1}+\zeta_{i}^{*} \\
= & \pi_{i} I_{i}+\pi_{i} \gamma_{i}^{*}\left(1 / \pi_{d}\right) \text { Dummy }+\pi_{i} \xi_{i}^{*} \\
& \quad \text { since } \xi_{1}=\left(1 / \pi_{d}\right) \text { Dummy. }
\end{aligned}
$$

Then, we have the following equations:

$$
\begin{aligned}
& \gamma_{i+3}=\pi_{i} I_{i}, \\
& \gamma_{i}=\left(1 / \pi_{d}\right) \gamma_{i}^{*} \pi_{i}, \\
& \zeta_{i}=\pi_{i} \zeta_{i}^{*} \quad \text { for } i=1,2,3 .
\end{aligned}
$$


Note that all the parameters in the LISREL model are functions of the parameters of the PLS model. For example, $\gamma_{i}$, the mean difference parameter for $Y_{i}$ in the LISREL model, can be calculated by $\left(1 / \pi_{d}\right) \gamma_{i}^{*} \pi_{i}$ from the PLS solutions. That is, the models are equivalent in terms of specification. However, important differences exist with respect to estimation, the properties of estimators, test statistics, and related issues which dictate the choice of the model. These issues are discussed below.

\subsection{An illustration}

Bagozzi and Yi's (1989) data are used to illustrate the equivalence of results using the PLS analysis and results from the LISREL analysis. Specifically, the three behavior measures are used as the dependent variables in MANOVA designs with two groups (see Bagozzi and Yi, 1989, and our Table 1 and Fig. 1). Table 1 reports the means, standard deviations, and correlations for the input data. 'The LISREL solutions are obtained by using the LISREL VI program (Jöreskog and Sörbom, 1986). The PLS model is estimated with the LVPLS 1.6 program (Lohmöller, 1984), and critical ratios for the PLS estimates are calculated by the jackknifing of parameter estimates (Efron and Gong, 1983). Specifically, LVPLX was employed because we needed to estimate location parameters, and option 4 was selected for the data metric. Appendix A provides the specification for the PLS model in Fig. 1. Standard errors of parameters were estimated by using the jackknife procedures which were

Table 1

Data for LISREL and PLS models

\begin{tabular}{lllll}
\hline Measure & $n=152$ & & & \\
\hline Behavior 1 & 1.000 & & & \\
Behavior 2 & 0.689 & 1.000 & & \\
Behavior 3 & 0.658 & 0.941 & 1.000 & \\
Dummy & 0.424 & 0.481 & 0.508 & 1.000 \\
Mean & 2.204 & 1.013 & 2.388 & 0.520 \\
Standard deviation & 4.456 & 1.483 & 3.491 & 0.501 \\
\hline
\end{tabular}

Table 2

MANOVA results for LISREL and PLS models

\begin{tabular}{llrr}
\hline & LISREL model & \multicolumn{1}{l}{ PLS model } & \\
\hline Mean differences & $\gamma_{1}=3.84(5.7)^{a}$ & $\gamma_{1}^{*}=0.424$ & $(10.3)$ \\
& $\gamma_{2}=1.42(6.7)$ & $\gamma_{2}^{*}=0.481$ & $(9.0)$ \\
& $\gamma_{3}=3.54(7.2)$ & $\gamma_{3}^{*}=0.508$ & $(9.2)$ \\
Other parameters & $\gamma_{4}=0.20(0.4)$ & $\pi_{1}=4.531$ & $(13.7)$ \\
& $\gamma_{5}=0.27(1.8)$ & $\pi_{2}=1.478$ & $(20.0)$ \\
& $\gamma_{6}=0.55(1.6)$ & $\pi_{3}=3.479$ & $(22.9)$ \\
& & $\pi_{d}=0.500(312.7)$ \\
& & $I_{1}=0.045$ \\
& & $I_{2}=0.185$ \\
& & $I_{3}=0.157$ \\
\hline
\end{tabular}

${ }^{a}$ Critical ratios are given in parentheses.

developed by Barclay (1983) (see, e.g., Fenwick, 1979; Cooil, Winer and Rados, 1987). Table 2 summarizes the key results from the LISREL and PLS analyses.

The full LISREL model specified in Fig. 1A, which allows for the differences in means, is exactly identified and gives a perfect fit to the data: $\chi^{2}(0)=0.00, p=1.00$. The restricted model with the zero constraints for the mean difference parameters (i.e., $\gamma_{1}=\gamma_{2}=\gamma_{3}=0$ ) gives the following results: $\chi^{2}(3)=48.21, p$ $<0.001$. An omnibus test of mean difference can be conducted by comparing the fit of these two models. The significant chi-square difference $\left(\chi_{d}^{2}(3)=48.21, p<0.001\right)$ suggests that the means on at least one dependent variable are significantly different across groups. The estimates for individual mean difference parameters are examined to test whether each dependent variable is different across groups. The mean differences, denoted as $\gamma_{i}$ in the LISREL analysis, are $3.84(t=5.2)$, $1.42(t=6.7)$, and $3.54(t=7.2)$, respectively. They are all significant, suggesting that the means of all dependent variables are significantly different across groups for these particular data.

On the other hand, the PLS model in Fig. 1B gives the following results: $\gamma_{1}^{*}=0.424$ $(t=10.3), \quad \gamma_{2}^{*}=0.481 \quad(t=9.0), \quad$ and $\gamma_{3}^{*}=$ $0.508(t=9.2)$. These results suggest the same conclusion: the means of the three dependent 
variables are significantly different between the two groups. In fact, the solutions for the LISREL analysis can be calculated from the solutions from the PLS analysis. For example, the mean difference for $Y_{1}$ can be computed as $\left(1 / \pi_{d}\right) \gamma_{1}^{*} \pi_{1}=2.00 \times 0.42 \times 4.53=3.84$. Note that this value is identical to the estimate of $\gamma_{1}$ in the LISREL model. Similarly, $\gamma_{2}$ and $\gamma_{3}$ can be calculated from the PLS solutions. Also, $\gamma_{4}-\gamma_{6}$ can be obtained from the PLS solutions: $\gamma_{i+3}=\pi_{i} I_{i}$. For example, the mean $\left(\gamma_{4}\right)$ of $Y_{1}$ for the control group can be obtained by $\pi_{1} I_{1}=4.53 \times 0.045=0.20$.

\section{Pls models for various MANOVA designs}

We have shown that PLS can be used to analyze experimental data with an example of the basic Manova design. However, PLS is applicable not only to the basic design, but also to other MANOvA designs (e.g., latent variable MANOVA, step-down analysis, MANCOVA), which were discussed by Bagozzi and Yi (1989). In this section, we will examine some of these MANOVA designs and illustrate the application of PLS models. Because these extensions are rather straightforward, they are discussed briefly in this paper. However, the full results and corresponding specifications are available from the authors.

\subsection{Latent variable MANOVA}

Bagozzi and Yi (1989) extended the structural equation approach to MANOVA on latent variables, whereas the traditional MANOVA analysis is conducted only at the level of manifest variables (observed measures). This extension is motivated by several considerations (Bagozzi and Yi, 1989, pp. 273-274). First, if individual measures of the variables show excessive random error, the traditional tests may be lacking in statistical power to detect valid experimental effects. Second, certain variables might be inherently unobservable constructs such that they can be measured only indirectly with multiple indicators. Third, one might be concerned more with explanation and understanding of latent variables or constructs than with prediction or description of observed variables or measures per se.

\section{A. LISREL Specification}

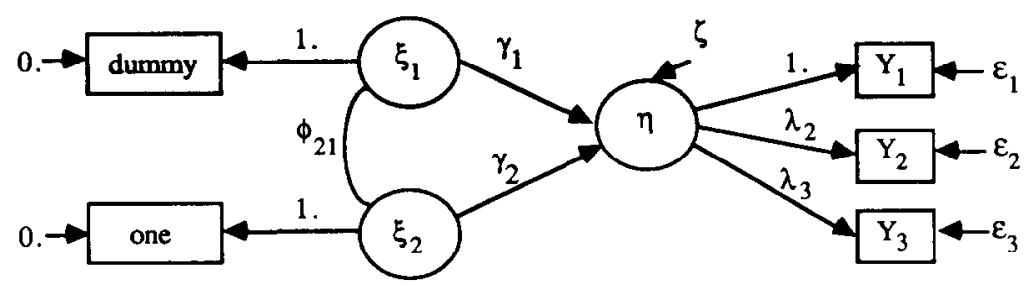

B. PLS Specification

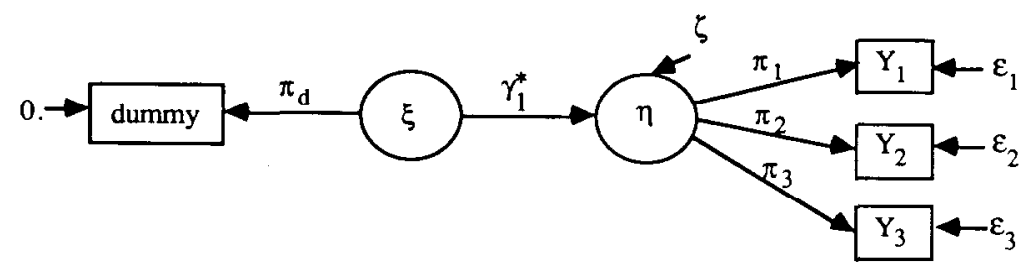

Fig. 2. LISREL and PLS models for MANOVA on three measures of a latent variable. 
Fig. 2A presents the LISREL specification for a latent variable MANOVA design appropriate to the data in Table 1 . Note that three behavioral measures are used here as multiple indicators of a single latent variable. We wish to test whether the experimental manipulation affects the mean of the theoretical construct as measured by three indicators. The path $\left(\gamma_{1}\right)$ from the dummy variable $\left(\xi_{1}\right)$ to the latent dependent variable $(\eta)$ reflects the difference in the means of the behavioral construct. When the LISREL model is fit to the data in Table 1, the estimate of $\gamma_{1}$ is 3.21 with $t=6.43$. These results show that the means of $\eta$ are significantly different across the two groups.

Fig. 2B provides a diagram of the corresponding PLS model for the same data. We note that the PLS specification is quite similar to the LISREL specification. One difference concerns the pseudo-variable of "One". In the LISREL model, the pseudo-variable of "One" is introduced to estimate the means for the control group. In contrast, introducing such a pseudo-variable is not necessary in the PLS model, where location parameters can be estimated directly. When the PLS model in Fig. 2B is fit, the estimate of $\gamma_{1}^{*}$ is 0.51 with $t=11.0$, suggesting the rejection of the null hypothesis of equal means for the two groups. Thus, both LISREL and PLS give the same conclusion in the latent variable MANOVA.

Remark. One can show the equivalence of the IISREL and PLS models as follows:

LISREL model:

$$
\begin{aligned}
Y_{i} & =\lambda_{i} \eta+\varepsilon_{i} \\
& =\lambda_{i}\left(\gamma_{1} \xi_{1}+\gamma_{2} \xi_{2}+\zeta\right)+\varepsilon_{i} \\
& =\lambda_{i} \gamma_{2}+\lambda_{i} \gamma_{1} \text { Dummy }+\lambda_{i} \zeta^{*}+\varepsilon_{i}^{*} . \\
& P L S \text { model: } \\
Y_{i} & =\pi_{i} \eta+\varepsilon_{i}^{*} \\
& =\pi_{i}\left(I+\gamma_{1}^{*} \xi_{1}+\zeta^{*}\right)+\varepsilon_{i}^{*} \\
& =\pi_{i} I+\pi_{i} \gamma_{1}^{*}\left(1 / \pi_{d}\right) \text { Dummy }+\pi_{i} \zeta^{*}+\varepsilon_{i}^{*} .
\end{aligned}
$$

Then, we have the following equations:

$\lambda_{i} \gamma_{2}=\pi_{i} I$,

$\lambda_{i} \gamma_{1}=\left(1 / \pi_{d}\right) \gamma_{1}^{*} \pi_{i}$,

$\lambda_{i} \zeta+\varepsilon_{i}=\pi_{i} \zeta^{*}+\varepsilon_{i}^{*} \quad$ for $i=1,2,3$.

Since all the parameters of the LISREL model are functions of the parameters in the PLS model, the models are equivalent in terms of specification.

\subsection{Step-down analysis}

Bagozzi and Yi (1989, pp. 274-276) describe and illustrate step-down analyses with structural equation models. When there is a causal order among the dependent variables, step-down analyses provide useful information as to whether the mean difference in a certain variable is due to the direct effect of the experimental manipulation or its dependence on other variables (Roy and Bargmann, 1958). The first stage of a step-down analysis begins with a MANOVA test performed on all dependent variables. If the omnibus test points to a rejection of equal means, then the next step consists of testing the final variable in the hypothesized causal chain while partialling out all remaining dependent variables as covariates. A significant omnibus test would indicate that the final variable differs even after controlling for its dependence on previous variables. In contrast, a non-significant test suggests that the difference in the final criterion is wholly due to the causal relation between the final variable and other variables.

Fig. 3 shows the step-down analysis procedures for the MANOVA design with two latent dependent variables (i.e., decision and behavior), which has been illustrated by Bagozzi and Yi (1989). In Step One, the mean differences in decision $(D)$ and behavior $(B)$ are tested while their covariation is unexplained. In the next step, the difference in $B$ is tested while controlling for the causal path from $D$ 


\section{A. Step One}

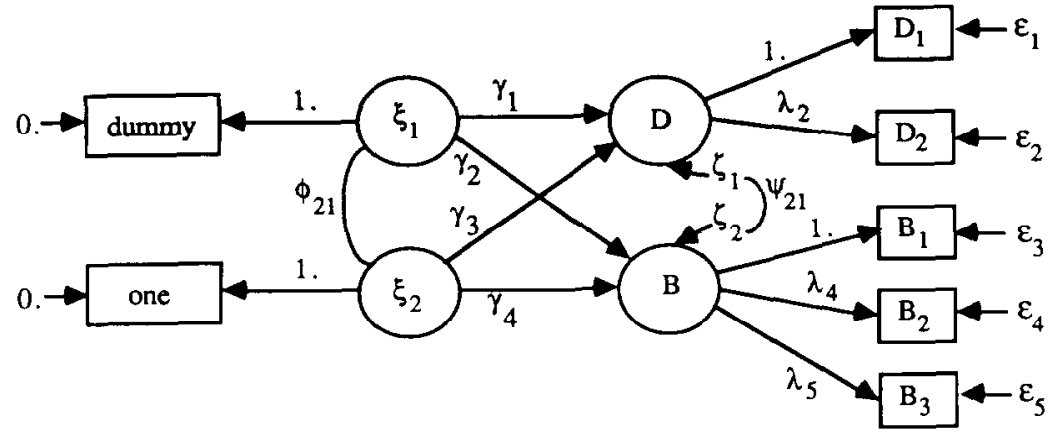

B. Step Two

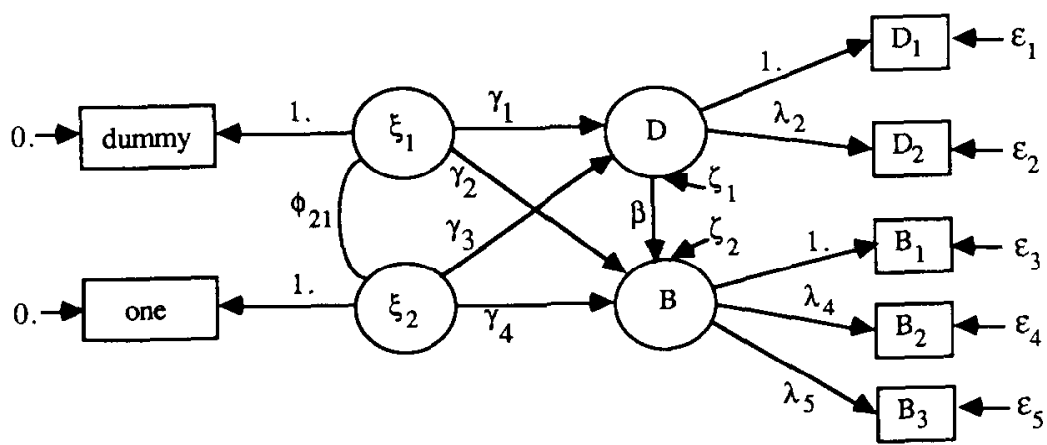

Fig. 3. Step-down analysis with two latent variables: dummy variable approach.

to $B$. This procedure is called the dummy variable approach, because an indicator variable is used to test the mean difference across groups.
Pls can also be applied to step-down analysis. The LISREL specifications for step-down analysis for the data, which were originally illustrated by Bagozzi and Yi (1989), are pre-

Tablc 3

Data for step-down analyses

\begin{tabular}{|c|c|c|c|c|c|c|c|c|c|c|}
\hline Measure & High & dance & $\mathrm{up}(n=$ & & & Low in & dance & $p(n=$ & & \\
\hline Behavior 1 & 1.000 & & & & & 1.000 & & & & \\
\hline Behavior 2 & 0.774 & 1.000 & & & & 0.641 & 1.000 & & & \\
\hline Behavior 3 & 0.736 & 0.945 & 1.000 & & & 0.580 & 0.921 & 1.000 & & \\
\hline Decision 1 & 0.256 & 0.425 & 0.430 & 1.000 & & 0.255 & 0.173 & 0.171 & 1.000 & \\
\hline Decision 2 & 0.263 & 0.426 & 0.430 & 0.907 & 1.000 & 0.263 & 0.205 & 0.181 & 0.882 & 1.000 \\
\hline Mean & 0.206 & 0.274 & 0.548 & 4.027 & 3.932 & 4.050 & 1.696 & 4.089 & 4.899 & 4.760 \\
\hline Standard deviation & 0.726 & 0.838 & 1.633 & 1.462 & 1.456 & 5.686 & 1.620 & 3.877 & 1.446 & 1.398 \\
\hline
\end{tabular}


sented in Fig. 3. Table 3 reports the means, standard deviations, and within-group correlations for the input data. The corresponding PLS specifications can easily be obtained by dropping the pseudo-variable of "One" and the latent variable associated with it (see Figs. 1 and 2 for examples). In Step One, Manovas are conducted on the two latent variables ( $D$ and $B$ ) which are measured with several indicators. The LISREL model gives the following results: $\gamma_{1}=0.86(t=3.8)$ and $\gamma_{2}=3.20(t=$ 6.4). The PIS model gives the following results: $\gamma_{1}^{*}=0.29(t=3.4)$ and $\gamma_{2}^{*}=0.51(t=$ 11.0). Thus, both LISREL and PLS results suggest that the mean differences for $D$ and $B$ are statistically significant.

In Step Two, MANOVAs are conducted while controlling for the causal relation $(\beta)$ between $D$ and $B$ so that the effect of $D$ on $B$ can be partialled out. The LISREL model gives the following results: $\gamma_{1}=0.86(t=3.8), \gamma_{2}=$ $2.74(t=5.6)$, and $\beta=0.54(t=3.21)$. The PLS model gives the following results: $\gamma_{1}^{*}=$ $0.29(t=3.4), \gamma_{2}^{*}=0.44(t=9.5)$, and $\beta^{*}=$ $0.23(t=4.3)$. The results show that $D$ has a significant effect on $B$, as hypothesized. The results also show that the two groups differ significantly in $B$ even after controlling for its dependence on $D$. In sum, both LISREL and PLS can be applied to step-down analysis, and they give the same conclusions.

\subsection{Homogeneity and multiple-group approach}

In the previous example of step-down analysis, we have employed the dummy variable approach in both LISREL and PLS analyses. However, the dummy variable approach assumes that variances and covariances of dependent variables are equal across groups. This assumption is imposed because the covariance matrices among dependent variables are collapsed into one (i.e., the submatrix of the covariance matrix after dropping the dummy variable column) under the dummy variable approach. The homogencity assump- tion is made implicitly under the dummy variable approach, whether one employs LISREL or PLS.

When the homogeneity assumption is violated, the multiple group approach can be used instead (see Remark below). As we will see in the next section of this paper, for instance, LISRLL can be used for the multiple group approach to step-down analysis. Then, a natural question would arise: can PLS be applied to the multiple group approach (e.g., for step-down analysis)? Unfortunately, multiple group (simultaneous) analysis, which is needed for the multiple group approach to MANOVAs, is not available at this time for PLS. ${ }^{1}$ Thus, although PLS can be applied to various MANOVA designs such as manifest variable MANOVA, latent variable MANOVA, and step-down analysis, it cannot be applied to the multiple group approach.

Remark. A reviewer suggested that when the homogeneity assumption is violated, two other alternatives than the multiple group approach can be used. One alternative can be used when variances are heterogeneous. In such a case, one can identify which variable shows heterogeneous variance (e.g., via Cochran test) and use transformations to stabilize the variance. When the relationship between the mean and variance is known, one can find a transformation of the variable, which makes the variance approximately constant. For example, if the standard deviation is proportional to the mean, one can used the logarithmic transformation. Or if the variable (e.g., $y$ ) follows the Poisson distribution, one can use $y^{1 / 2}$ or $y^{1 / 2}+(y+1)^{1 / 2}$. The resulting variance will be constant (see Bartlett, 1947, or Kendall and Stuart, 1968, pp. 88-92, for more details). On the other hand, when the rela-

1 Some effort is currently being made to develop formal PLS procedures for the simultaneous analysis of multiple sample, but they are not yet available (Fornell, 1990, personal communication), although it is impossible to say at this time when, or even if, the effort will bear fruit. 
tionship between the mean and variance is unknown, one can examine the Box-Cox diagnostic plot to select the appropriate transformation (Box and Cox, 1964) (see the BMDP manual, 1988, pp. 210-211, for an example).

A second alternative is appropriate when covariances are heterogeneous. One can give all measurement variables unit variance during the estimation and rescale the loadings afterwards to the original metric, which is implemented in the PLS program (metric $=3$ ). This results in a communality maximization described in Lohmöller's program. According to the reviewer, this procedure works well in practice when multivariate normality as well as homogeneity of covariance matrices are not met.

\section{Multiple group approach to step-down analysis}

Fig. 3 shows the step-down analysis procedures for the MANOVA design with two latent dependent variables, which has been illustrated by Bagozzi and Yi (1989). This procedure is called the dummy variable approach, because an indicator variable is used to test the mean difference across groups.

The dummy variable approach to stepdown analysis, however, makes two implicit assumptions. First, it assumes that the variances and covariances of dependent variables are equal across groups. This is also a standard assumption in traditional MANOVA analyses (e.g., BMDP, SAS, SPSS $^{\mathrm{X}}$ ). Second, the causal relations among dependent variables are assumed to be invariant across groups. That is, the effects of one variable on other variables are assumed to be identical for all groups. This assumption is also implicitly made in traditional analyses (e.g., sPss ${ }^{\mathrm{X}}$ ). It is not likely that these assumptions are valid for all manova designs. To the extent that these assumptions are violated, the procedures suggested by Bagozzi and Yi (1989) could be misleading. ${ }^{2}$ It seems desirable to consider an alternative procedure which does not make such restrictive assumptions. At least, it is necessary to make such assumptions explicit and test whether they are valid or not in any particular application.

In this regard, we suggest a multiple group approach to step-down analyses. Fig. 4 shows the general procedure. In Step One, $\gamma_{1}$ and $\gamma_{2}$ correspond to the means of latent variables $D$ and $B$, respectively. Thus, the equality of means can be tested by comparing these parameters (i.e., $\gamma_{i}^{(1)}$ vs. $\gamma_{i}^{(2)}$ ) across groups. This can be accomplished via a simultaneous analysis of both groups. In Step Two, $\gamma_{2}$ would correspond to the portion of the mean for $B$ that is not due to the effect of $D$. Thus, a comparison of $\gamma_{2}$ across groups indicates whether the means of $B$ differ between the group when the effect of $D$ on $B$ is partialled out.

One advantage of this approach is that it allows one to test the aformentioned assumptions, i.e., (1) homogeneity of variances and covariances, and (2) invariance of causal paths. Specifically, before conducting the first step of the analysis noted in Fig. 3, one can test the homogeneity assumption in the multiple group approach. Then, in Step One the differences in $D$ and $B$ can be tested under either homogeneity or heterogeneity assumptions. One can also test the invariance of causal paths (i.e., $\beta^{(1)}=\beta^{(2)}$ ) across groups. If this test is significant, a subsequent step would be to test for the significance of the

2 It should be acknowledged that Bagozzi and Yi (1989) noted that the dummy variable approach assumes homogeneity like the traditional MANOVA analyses. They also considered a multiple group approach to other MANOva designs which can handle violations of the homogeneity assumption. However, their procedures did not explicitly address the homogeneity assumption in step-down analyses. Furthermore, the invariance of causal paths was not mentioned in Bagozzi and Yi's (1989) paper. As a consequence, there is some potential for misunderstanding among readers. This paper attempts to clarify these issues by explicitly pinpointing these implicit assumptions and illustrating the consequences of violating the assumptions. 


\section{A. Step One}

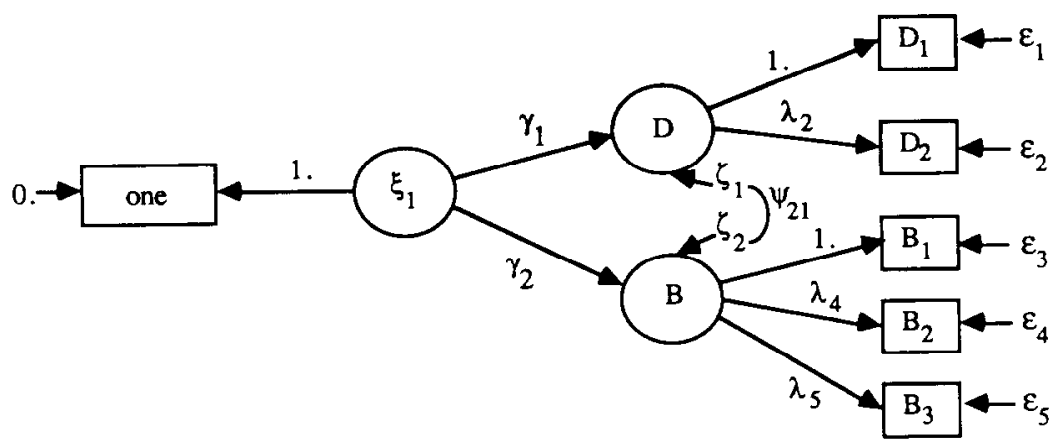

B. Step Two

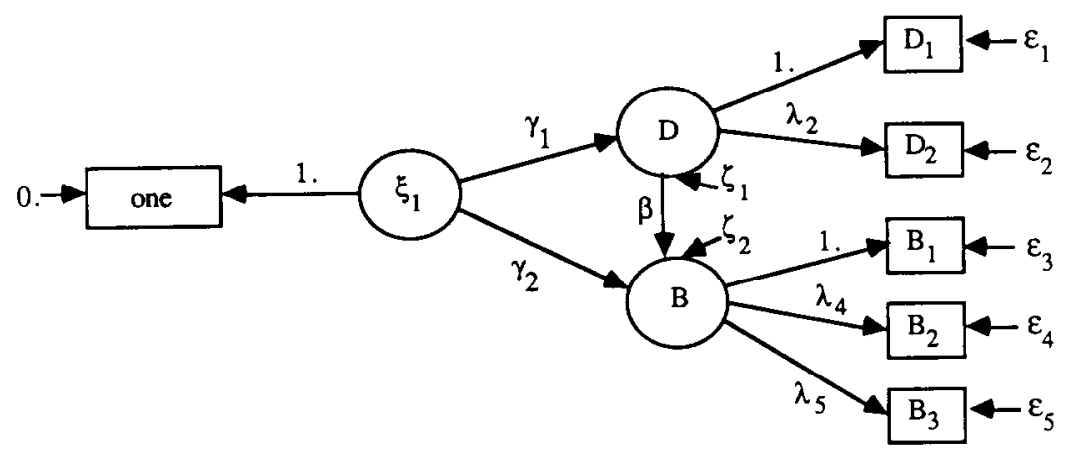

Fig. 4. Step-down analysis with two latent variables: multiple-group approach.

mean difference while allowing for different causal paths across groups. Thus, another advantage of the multiple group approach is that it allows for step-down analyses even when these assumptions are violated.

\subsection{An illustration}

The suggested procedures for step-down analyses are illustrated with the example used by Bagozzi and Yi (1989). There are two latent variables: decision $(D)$ and behavior $(B)$, which are measured with two $\left(d_{1}, d_{2}\right)$ and three indicators $\left(b_{1}, b_{2}, b_{3}\right)$, respectively (see Tables 4 and 5 for a summary of the results from Bagozzi and Yi's, 1989, procedures and the suggested procedures, respectively).
Results from the dummy variable approach are examined first. In Step One, the mean difference are $0.86(t=3.8)$ and $3.20(t=6.4)$ for $D$ and $B$, respectively. The chi-square difference test also indicates that the mean differences are statistically significant; $\chi_{d}^{2}(2)$ $=45.97, p<0.001$. In Step Two, one can test the mean difference in $B$ after considering the causal order between $D$ and $B$. The chisquare difference test indicates that the mean difference in the final dependent variable (i.e., $B)$ is significant; $\chi_{d}^{2}(1)=31.69, \quad p<0.001$. Thus, the two groups still differ significantly in $B$ even after considering its dependence on $D$.

Next, the multiple group approach is used. We begin by testing the homogeneity of variance and covariances across groups. This test 
Table 4

Step-down analysis with a dummy variable approach

\section{First stage}

\begin{tabular}{ll} 
Full model & Restricted model with $\gamma_{1}=\gamma_{2}=0$ \\
\hline$\chi^{2}(10)=7.88$ & $\chi^{2}(12)=53.85$ \\
$p \approx 0.64$ & $p \approx 0.000$ \\
$\gamma_{1}=0.86(3.76)^{a}$ & Hence: $\chi_{d}^{2}(2)=45.97, p \approx 0.000$ \\
$\gamma_{2}=3.20(6.42)$ &
\end{tabular}

\section{Second stage}

\begin{tabular}{ll} 
Full model & Restricted model with $\gamma_{2}=0$ \\
\hline$\chi^{2}(10)=7.88$ & $\chi^{2}(11)=39.57$ \\
$p \approx 0.64$ & $p \approx 0.000$ \\
$\gamma_{2}=2.74(5.56)$ & Hence: $\chi_{d}^{2}(1)=31.69, p \approx 0.000$ \\
\hline
\end{tabular}

Critical ratios are given in parentheses.

is conducted by comparing the model with free covariance matrices and the model with equal covariance matrices for residuals. The

Table 5

Step-down analysis with a multiple group approach

\section{Homogeneity test}

\begin{tabular}{ll} 
Full model & $\begin{array}{l}\text { Restricted model with } \\
\text { equal variances }\end{array}$ \\
\hline$\chi^{2}(0)=0.00, p \approx 1.00$ & $\begin{array}{l}\chi^{2}(15)=351.96, p \approx 0.000 \\
\text { Hence: } \chi_{d}^{2}(15)=351.96, \\
p \approx 0.000\end{array}$
\end{tabular}

\section{First stage}

Full model

Restricted model with $\gamma_{1}^{(1)}=\gamma_{2}^{(2)}$

$\chi^{2}(14)=7.89, p \approx 0.90 \quad \chi^{2}(16)=66.08, p \approx 0.000$

$\gamma_{1}^{(1)}=4.03(0.17)^{\mathrm{a}}$

$\gamma_{1}^{(2)}=4.90(0.16)$

$\gamma_{2}^{(1)}=0.19(0.07)$

$\gamma_{2}^{(2)}=4.01(0.55)$

Invariance of causal path test

Full model Restricted model with $\beta^{(1)}=\beta^{(2)}$

$\chi^{7}(14)=7.89, p=0.90 \quad \chi^{7}(15)=9.35, p \approx 0.86$

$\beta^{(1)}=0.18(0.05) \quad$ Hence: $\chi_{d}^{2}(1)=1.46$,

$\beta^{(2)}=0.58(0.32)$ $p>0.10$

Second stage

Full model

Restricted model with $\gamma_{2}^{(1)}=\gamma_{2}^{(2)}$

$\chi^{2}(15)=9.35, p \approx 0.86 \quad \chi^{2}(16)=60.58, p \approx 0.000$

$\gamma_{2}^{(1)}=-0.58(0.19)$

Hence: $\chi_{d}^{2}(1)=51.23$,

$\gamma_{2}^{(2)}=3.04(0.59)$ $p \approx 0.000$

\footnotetext{
"Standard errors are given in parentheses.
}

results indicate that the homogeneity assumption should be rejected for the data; $\chi_{d}^{2}(15)=$ $351.96, p<0.001$. Thus, the subsequent analyses are conducted while allowing for different variances and covariances for the two groups.

When the mean parameters $\left(\gamma_{i}\right.$ 's) are allowed to differ across groups, the model gives satisfactory results: $\chi^{2}(14)=7.89, p>0.89$. The estimates of mean parameters for both groups are 4.03 and 4.90 for $D$, and 0.19 and 4.01 for $B$, respectively. When the mean parameters are fixed to be invariant across groups, the model fit is not satisfactory; $\chi^{2}(16)=66.08, p<0.001$. The chi-square difference is 58.19 with 2 degrees of freedom, which is significant at the 0.001 level. Thus, the equality constraints (i.e., $\gamma_{i}^{(1)}=\gamma_{i}^{(2)}$ for $i=1,2$ ) produce a significant increase in the chi-square values, suggesting the rejection of the null hypothesis that means are equal across groups.

Before moving to the second stage, the invariance of the causal path (i.e., $\beta^{(1)}=\beta^{(2)}$ ) is tested by comparing the full model without the equality constraint and the restricted model with the constraint. The full model without the constraint gives the following results: $\chi^{2}(14)=7.89, p>0.89$. The restricted model with the constraint yields the following result: $\chi^{2}(15)=9.35, p>0.85$. The chi-square difference is 1.46 with 1 degree of freedom, which is not significant at the 0.10 level. One cannot reject the hypothesis that the causal path between $D$ and $B$ is invariant across groups. That is, the assumption of invariant causal paths is plausible for this data set. Subsequent analyses are thus conducted using the invariant causal path in the model.

The next step examines the equality of means in $B$ while controlling for the effect of $D$ (see Step Two in Fig. 3). The model allowing for different means of $D$ shows satisfactory results: $\chi^{2}(15)=9.35, p>0.95$, whereas the restricted model hypothesizing equal means for $D$ reveals unsatisfactory results: 
$\chi^{2}(16)=60.58, p<0.001$. The chi-square difference is 51.23 with 1 degree of freedom, which is significant at the 0.001 level. These findings suggest that the hypothesis of equal means for behavior be rejected even after controlling for the effect of decision.

Note that this chi-square difference test $\left(\chi_{d}^{2}(1)=51.23\right)$ is different from that $\left(\chi_{d}^{2}(1)\right.$ $=31.69)$ in the dummy variable approach (see Table 4). This illustrates that the multiple group approach and the dummy variable approach differ in handling the two assumptions. In this example, since we found that the homogeneity assumption is not valid, the multiple group approach is conducted while allowing for heterogeneous variances and covariances. In contrast, the dummy variable approach analyzes the data as if the variances and covariances were equal across the groups when in fact they are not. Thus, the two approaches can yield different results. Although the final conclusions happen to be the same in this particular case, the two approaches could suggest different conclusions in other cases.

\section{Discussion}

We have seen that both LISREL and PLS models can be used to analyze experimental data. The question arises: under what conditions should one model be preferred to the other? A comparison of estimation methods between the two models would be useful in this regard (Fornell and Bookstein, 1982; Jöreskog and Wold, 1982). PLS, which uses fixed point estimation (see, e.g., Wold, 1965), differs from LISREL which uses maximum likelihood (ML) estimation in its basic assumptions and principles. The ML estimation in LISREL maximizes the probability of observing the data given the hypothesized model assuming interval scales and multivariate normality of variables. However PLS uses a series of interdependent oLs regressions to minimize residual variances without making any assumptions with respect to the population or scales of measurement. Hence, no distributional assumptions are required. The PLS procedure is also applicable even when the sample size is small. Wold (1986) reports an analysis with a sample of 10 , and Fornell and Bookstein (1982) use PLS on a sample of 24. In the former study, 28 manifest variables were included in the model. Analyses of such data sets by maximum likelihood procedures are often not feasible (Wold, 1989). Sampling errors or too many parameters to estimate can yield non-convergent and improper solutions in LISREL analyses, which make it difficult to interpret the solutions (see, e.g., Gerbing and Anderson, 1987). In contrast, PLS does not suffer from non-convergent or improper solutions (Fornell and Bookstein, 1982).

An examination of the preceding assumptions suggests that the use of PLS is preferred over LISREL when (1) the multivariate normality assumption is violated, (2) the sample size is small, and (3) non-convergent or improper solutions are likely to occur (e.g., a complex model with many parameters). A reviewer noted that the second situation (small sample size) is the most important one in experimental designs. The assumption of multivariate normality can be relaxed with elliptical estimation or asymptotic distribution-free estimation (see, e.g., Browne, 1984), but this requires a large sample size (typically, the sample size must be 200 or more, depending on the number of variables in the model). Also, non-convergent or improper solutions are less likely to occur for large sample sizes (see, e.g., Anderson and Gerbing, 1984). Nevertheless, obtaining a large sample size might be difficult in typical experimental designs.

Some problems of the PLS approach also need to be mentioned. First, PLS tends to overestimate loadings and underestimate path coefficients (Dijkstra, 1983). In fact, as the proposed methodology is primarily concerned 
with path coefficients (which are underestimated), the significant results in a PLS analysis can be given more credence, because the test would be more conservative. ${ }^{3}$ Another problem with PLS concerns the interpretation of parameter estimates. ${ }^{4}$ The substantive interpretation of LISREL estimates is clear. In Fig. 1A, for example, $\gamma_{1}-\gamma_{3}$ correspond to the mean differences of dependent variables across the control and experimental groups, whereas $\gamma_{4}-\gamma_{6}$ reflect the means of dependent variables for the control group. In contrast, the parameter estimates in the PLS specification do not have such direct interpretations. Rather, they are multiplicative components of the means or mean differences, as shown earlier. Still another problem with PLS applications is that jackknife or bootstrap procedures are needed to obtain estimates for the standard errors of the parameter estimates, which are potentially subject to biases (Dijkstra, 1983; Efron and Gong, 1983). Furthermore, because it is a limited-information estimation method, PLS parameter estimates are not as efficient as full-information estimates (Fornell and Bookstein, 1982). Finally, PLS does not provide formal statistical tests or multiple sample analysis procedures, which are available for LISREL.

We have also shown that the dummy variable approach to step-down analysis makes two assumptions: (1) homogeneity of variances and covariances, and (2) invariance of causal paths. The homogeneity assumption is often violated and its violation can have serious consequences especially when the sample size is unequal across groups (see, e.g., Bray and Maxwell, 1985; Kühnel, 1988). Indeed, we have seen that this assumption is rejected for the data used in Bagozzi and Yi's (1989) step-down analyses. The second assumption can also be problematic, because experimen-

\footnotetext{
3 We thank a reviewer for bringing this point to our attention.

4 We thank a reviewer for pointing out to us this problem with PLS.
}

tal manipulations are often designed to influence causal paths among variables, as well as their means.

The multiple group approach to step-down analysis, which is proposed in this article, does not make these restrictive assumptions. Instead, it tests these and provides information regarding how reasonable the two assumptions are. Further, it allows for stepdown analysis even when these assumptions are violated. However, the multiple group approach has several limitations that deserve mention. It requires a relatively large sample size, because the sample is divided into experimental groups. If the example size is too small for each group, improper solutions and non-convergence might occur, a greater chance of making a Type II error exists, and asymptotic properties of the estimation are not obtained (see, e.g., Bearden, Sharma and Teel, 1982). Thus, the multiple group approach seems useful for step-down analysis when (1) variances and sample sizes are unequal across groups, (2) the experimental manipulation influences the theoretical relations among the dependent variables, and (3) the sample size is large enough for each group.

In this article, we have extended the use of structural equation models in experimental designs with respect to estimation methods in general and step-down analysis in particular. The analysis can be accomplished via PLS, which can be used even when certain assumptions for LISREL do not hold. We have also proposed a step-down analysis procedure which can be used even when the data do not meet the two restrictive assumptions implicit in Bagozzi and Yi's (1989) procedures.

Given the two extensions, a question arises naturally: can PLS be applied to the multiple group approach to step-down analysis? Unfortunately, the answer is no at this point in time. A multiple sample analysis, which is necessary for the multiple group approach, is not available for PLS. Thus, PLS cannot be used for the multiple group approach to 
MANOVA designs in general and step-down analysis in particular. Such procedures should be developed in future research.

Still more extensions need to be made to the structural equation approach to experimental data. For example, one should investigate more complex design issues such as multiple factors and levels. Such extensions will provide researchers with useful insights for making better applications of structural equation models in experimental designs.

\section{Appendix A. PLS specification for Fig. 1}

Number of blocks $=4$.

Number of cases $=152$.

Number of dimensions $=0$.

Output quantity $=3377$.

Inner weighting scheme $=1$.

Number of iterations $=100$.

Estimation accuracy $=5$.

Analyzed data metric $=4$.

${ }^{*}$ Read Matrix, Unit $=0$, Rewind $=0$, Format $=(2 \mathrm{~A} 4,4 \mathrm{~F} 2.0)$.

\begin{tabular}{lllll}
\hline Block & N-MV & Deflate & Direction & Model \\
\hline ZAI1 & 1 & 0 & Outwards & Exogenous \\
ETA1 & 1 & 0 & Outwards & Endogenous \\
ETA2 & 1 & 0 & Outwards & Endogenous \\
ETA3 & 1 & 0 & Outwards & Endogenous \\
& 4 & \multicolumn{3}{c}{ Mode A }
\end{tabular}

Path design matrix

\begin{tabular}{lllll}
\hline & ZAI1 & ETA1 & ETA2 & ETA3 \\
\hline ZAI1 & 0.00 & 0.00 & 0.00 & 0.00 \\
ETA1 & 1.00 & 0.00 & 0.00 & 0.00 \\
ETA2 & 1.00 & 0.00 & 0.00 & 0.00 \\
ETA3 & 1.00 & 0.00 & 0.00 & 0.00 \\
\hline
\end{tabular}

* Read Matrix, Unit $=0$, Rewind $=0$, Format $=(2 \mathrm{~A} 4, \mathrm{~F} 5.1,19 \mathrm{X}, 3(\mathrm{~F} 5.1,1 \mathrm{X}))$.

* The matrix format is optional, because it is specific to each research design.

\section{References}

Anderson, J.C. and D.W. Gerbing, 1984. The effect of sampling error on convergence, improper solutions, and goodness-of-fit indices for maximum likelihood confirmatory factor analysis. Psychometrika 49, 155-173.

Bagozzi, R.P. and Y. Yi, 1989. On the use of structural equation models in experimental designs. Journal of Marketing Research 26, 271-284.

Barclay, D.W., 1983. Jackknifing in PLS. Unpublished working paper, The University of Michigan.

Bartlett, M.S., 1947. The use of transformations. Biometrics 3, 3952.

Bearden, W.O., S. Sharma and J.E. Teel, 1982. Sample size effects on chi square and other statistics used in evaluating causal models. Journal of Marketing Research 19, 425-430.

BMDP Statistical Software Manual, 1988. Berkeley, CA: University of California Press.

Box, G.E.P. and D.R. Cox, 1964. Analysis of transformations. Journal of Royal Statistical Society, Series B 26, 211-252.

Bray, J.H. and S.E. Maxwell, 1985. Multivariate analysis of variance. Beverly Hills, CA: Sage Publications.

Browne, M.W., 1984. Asymptotically distribution-free methods for the analysis of covariance structures. British Journal of Mathematical and Statistical Psychology 32, 62-83.

Cooil, B., R.S. Winer and D.L. Rados, 1987. Cross-validation for prediction. Journal of Marketing Research 24, 271-279.

Dijkstra, T., 1983. Some comments on maximum likelihood and partial least squares methods. Journal of Econometrics $22,67-90$.

Efron, B. and G. Gong, 1983. A leisurely look at the bootstrap, the jackknife, and cross-validation. The American Statistician $34,36-48$.

Fenwick, I., 1979. Techniques in market measurement: The jackknife. Journal of Marketing Rescarch 16, 410-414.

Fornell, C. and F.L. Bookstein, 1982. Two structural equation models: LISREL and PLS applied to consumer exit-voice theory. Journal of Marketing Research 19, 440-452.

Gerbing, D.W. and J.C. Anderson, 1987. Improper solutions in the analysis of covariance structures: Their interpretability and a comparison of alternative respecifications. Psychometrika 52, 99-111.

Jöreskog, K.G. and D. Sörbom, 1986. LiSREL vI: Analysis of linear structural relationships by maximum likelihood, instrument variables, and least squares methods, 4 th ed. Mooresville, IN: Scientific Software.

Jöreskog, K.G. and H. Wold (eds.), 1982. Systems under indirect observation: Causality, structure, prediction. Amsterdam: North-Holland.

Kendall, M.G. and A. Stuart, 1968. The advanced theory of statistics, Vol. 3. London: Charles Griffin.

Kühnel, S.M., 1988. Testing MANOva designs with LISREL. Sociological Methods \& Research 16, 504-523.

Lohmöller, J.B., 1984. LVPLS 1.6 program manual: Latent variables path analysis with partial least-squares estimation. Köln: Zentralarchiv für Empirische Sozialforschung, Universität zu Köln.

Roy, J. and R.E. Bargmann, 1958. Test of multiple independence and the associated confidence bounds. Annals of Mathematical Statistics 29, 491-503. 
Wold, H., 1965. A fixed-point theorem with econometric background, I-II. Archiv für Matematik 6, 209-240.

Wold, H., 1982. Systems under indirect observations using PLS. In: C. Fornell (ed.), A second generation of multivariate analysis, Vol. 2, 325-347. New York: Praeger.

Wold, H., 1985. Partial least squares. In: Encyclopedia of statistical sciences, Vol. 6, 581-591. New York: Wiley.

Wold, H., 1986. Factors influencing the outcome of economic sanctions. In: P. Ibarrda (ed.), Sixto Rios Honorary Volume, 325 338. Madrid: Conejo Superior de Investigaciences Cientificas.

Wold, H., 1989. Introduction to the second generation of multivariate analysis. In: H. Wold (ed.), Theoretical empiricism, VII-XL. New York: Paragon House.

$\mathrm{Yi}, \mathrm{Y}_{\text {., }}$ 1990. The effects of contextual priming in print advertisements. Journal of Consumer Research 17, 215-222. 